\title{
Stress-inducible and tissue-specific promoters in transgenic tomatoes
}

Smirnova O.G.*, Kochetov A.V.

Institute of Cytology and Genetics, SB RAS, Novosibirsk, Russia

*e-mail:planta@bionet.nsc.ru

Tomato is one of the most important crop plants. A genetic modification of tomato has continuously expanded in recent years to include enhanced tolerance to biotic and abiotic stresses and improved nutrition and tastes of the fruit. Promoter is an important part of the genetic construct, which largely determines the pattern of transcription of the transgene. Apart from a strong constitutive CaMV35S promoter, a toolbox of promoters with defined specificities is necessary for efficient expression of transgenes. More than 170 gene promoters of different plant species have been investigated for their stress-inducible, developmental- and tissue-specific expression in tomatoes. Pollen-specific promoters may be used for a hybrid seed production. RNAi silencing of $S$-adenosylmethionine decarboxylase genes under the control of tapetal-specific AtA9 promoter results in male sterility of tomato plants. PsEND1 and LeFRK4 promoters fused to a cytotoxic ribonuclease gene also produced efficient male-sterility in tomatoes. For fruit quality improvement, MdACO, PpACO, and AcMADS1 promoters may be used. Le2A11 promoter was used for hepatitis B virus large surface antigen and other target genes expression in tomato fruits. Over-expression of mouse ornithine decarboxylase gene under the control of Le2A11 fruit-specific promoter enhances fruit quality in tomato. Root-specific AtNRT2.1 and AtRB7 promoters were used to drive the expression of the antifungal NIC and Thi2.1 genes. Resulting tomato plants conferred enhanced resistance to Fusarium oxysporum and Ralstonia solanacearum. CYP97A29, DFR, FLS, NIK and PMEI promoters displayed different activity patterns in nematode-infected and uninfected transgenic hairy roots. Expression of $L e B A D H$ gene under control of its own promoter can increase salt tolerance without affecting plant growth. TGP database gathers information on constitutive, tissue-specific, and inducible promoters, the activity of which has been characterized in transgenic plants. The TGP database interface enables searching for promoters with certain characteristics. The TGP database allows to select promoters with the desired stress- and tissue-specific activities for transgene expression in tomatoes.

Acknowledgements: The work was supported by a budget project No. 0324-2019-0039. 